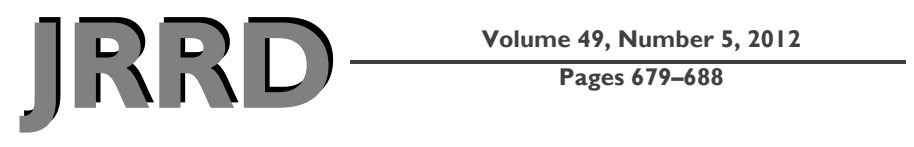

\title{
Review of exposure therapy: A gold standard for PTSD treatment
}

\author{
Sheila A. M. Rauch, PhD; ${ }^{*}$ Afsoon Eftekhari, PhD; ${ }^{2}$ Josef I. Ruzek, PhD $^{2-3}$ \\ ${ }^{1}$ Department of Veterans Affairs (VA) Ann Arbor Healthcare System, Ann Arbor, MI; and University of Michigan Medical \\ School, Ann Arbor, MI; ${ }^{2}$ National Center for PTSD, Dissemination and Training Division, VA Palo Alto Health Care \\ System, Palo Alto, CA; ${ }^{3}$ Stanford University, Stanford, CA; and Pacific Graduate School for Psychology, Palo Alto \\ University, Palo Alto, CA
}

\begin{abstract}
Prolonged exposure (PE) is an effective first-line treatment for posttraumatic stress disorder (PTSD), regardless of the type of trauma, for Veterans and military personnel. Extensive research and clinical practice guidelines from various organizations support this conclusion. PE is effective in reducing PTSD symptoms and has also demonstrated efficacy in reducing comorbid issues such as anger, guilt, negative health perceptions, and depression. PE has demonstrated efficacy in diagnostically complex populations and survivors of single- and multiple-incident traumas. The PE protocol includes four main therapeutic components (i.e., psychoeducation, in vivo exposure, imaginal exposure, and emotional processing). In light of PE's efficacy, the Veterans Health Administration designed and supported a PE training program for mental health professionals that has trained over 1,300 providers. Research examining the mechanisms involved in $\mathrm{PE}$ and working to improve its acceptability, efficacy, and efficiency is underway with promising results.
\end{abstract}

Key words: anger, cognitive behavioral therapy, combat, depression, exposure therapy, guilt, posttraumatic stress disorder, trauma, treatment, Veterans.

\section{INTRODUCTION}

In October 2010, the Department of Veterans Affairs (VA) and Department of Defense (DOD) released their updated VA/DOD Clinical Practice Guideline for Management of Posttraumatic Stress. In this update, exposure therapy (ET) remains a first-line treatment for posttrau- matic stress disorder (PTSD), with increased evidence supporting its use regardless of the type of trauma and comorbidities. In this article, we examine the evidence used to support this update to the guideline as well as review additional evidence of the effective use of ET with Veterans within the VA. Our discussion of ET will be focused on prolonged exposure (PE) because it is the most widely studied manualized version of ET for PTSD and is currently being disseminated in VA and DOD as a first-line intervention for PTSD.

ET has proven to be highly effective in reducing PTSD symptoms associated with a variety of traumas [1], including combat, noncombat, and military sexual trauma [2-5]. ET also significantly reduces comorbid symptoms that often occur with PTSD, including general

\footnotetext{
Abbreviations: $\mathrm{CBT}=$ cognitive behavioral therapy, $\mathrm{CSA}=$ child sexual abuse, DOD = Department of Defense, ET = exposure therapy, PCT $=$ present-centered therapy, $\mathrm{PE}=$ prolonged exposure, PPCS = prolonged postconcussive syndrome, PTSD = posttraumatic stress disorder, SSRI = selective serotonin reuptake inhibitor, $\mathrm{TBI}=$ traumatic brain injury, $\mathrm{VA}=$ Department of Veterans Affairs, VHA = Veterans Health Administration.

*Address all correspondence to Sheila A. M. Rauch, PhD; VA Ann Arbor Healthcare System, 2215 Fuller Rd (116c), Ann Arbor, MI 48105; 734-845-3545.

Email: sherauch@med.umich.edu
}

http://dx.doi.org/10.1682/JRRD.2011.08.0152 
anxiety, depression, guilt, and anger [6-7]. ET has repeatedly been supported as a first-line treatment for PTSD symptoms in treatment guidelines from various organizations [1], including the National Institute for Clinical Excellence [8] and the International Society for Traumatic Stress Studies [9]. An Institute of Medicine committee asserted that the only PTSD treatment with evidence "sufficient" to conclude its efficacy is ET [10]. Finally, in a recent VA/DOD expert consensus guideline for the treatment of patients with PTSD, mild traumatic brain injury (TBI), and pain, the panel concluded that, based on available research and clinical data, ET should remain a first-line treatment for PTSD among this complex and comorbid patient population [11]. Taken together, the effectiveness of ET is recognized across guidelines for the treatment of PTSD.

While the extensive background of research supporting the efficacy of ET for PTSD is apparent and began with studies among Veterans [2-4], Foa and Kozak developed the PE protocol using emotional processing theory almost 25 years ago [12]. This theory proposes that PE works through the processes of habituation, extinction, and emotional processing changes that occur in tandem with habituation (i.e., increased sense of competence, reduced sense of the world as dangerous) [13]. Foa and Kozak proposed that an effective psychosocial intervention for anxiety disorders requires modification of the pathological elements of a theoretical fear structure [12]. Specifically, they proposed two necessary conditions for modification of a fear structure: the fear structure must be activated, and information incompatible with the elements of the fear structure must be presented and integrated into the fear structure to replace pathological elements with more realistic ones. When applying emotional processing theory to PTSD, Foa and Riggs proposed two dysfunctional cognitions that are related to the development and maintenance of PTSD: "I am incompetent" (i.e., I cannot handle stress, my symptoms mean I am crazy), and "the world is completely dangerous" [14]. Through the process of repeated, prolonged confrontation of trauma-related stimuli, which occurs in $\mathrm{PE}$, habituation of emotional responses associated with the trauma occurs. As trauma-related stimuli are repeatedly confronted without the occurrence of feared consequences (e.g., going crazy, retraumatization) and with lessening emotional activation, the patient experiences repeated evidence that disconfirms dysfunctional cognitions specifically related to self-competence and control.
A stronger sense of self-competence and control over negative affect and stimuli then allows patients to reduce trauma-related avoidance, leading to even more spontaneous exposure and continued reduction of PTSD symptoms. Treatment-associated reductions in negative thoughts about the self and the world have been associated with symptom improvement [15], as has betweensession habituation of subjective anxiety during imaginal exposure [16-18]. Whether the emotional and cognitive changes are two aspects of a single process or whether one causes the other remains unclear.

The PE protocol includes four main therapeutic components (i.e., psychoeducation, in vivo exposure, imaginal exposure, and emotional processing). Psychoeducation occurs primarily in the first three sessions and focuses on PTSD symptoms and the patient's experience of those symptoms. Psychoeducation conveys the overarching idea that avoidance maintains PTSD symptoms, while confronting trauma-related stimuli and memories can reduce PTSD symptoms. In vivo exposure involves confronting people, places, and things that are trauma reminders. Imaginal exposure involves revisiting trauma reminders and engaging with the emotional content of the memory. During imaginal exposure, the patient closes his or her eyes and goes through the memory in the present tense with all the thoughts, feeling, and details he or she can include from the time of the trauma. Finally, emotional processing involves the therapist moving through the emotional content of the memory with the patient through open-ended discussion of the trauma and the experience of the exposures (i.e., review of changes in distress, review of progress in therapy, noting things that may have changed between sessions or memory repetitions). In addition, emotional processing also includes discussion about the patient's thoughts about him- or herself at the time of the trauma and now. This does not involve formal cognitive intervention or directive challenging but more open-ended discussion of the themes noted earlier.

\section{METHODS}

The following review of literature is based on the authors' (Rauch and Ruzek) participation in the extensive review of literature that occurred for the update in the VA/DOD PTSD clinical practice guidelines (2010). Additional relevant literature that has been published 
since this review, as well as personal communications with principal investigators on primary ongoing exposure therapy for PTSD clinical trials, is also included.

\section{RESULTS}

The PE protocol is quite amenable to dissemination and modification for alternate populations and settings. Indeed, PE has been successfully disseminated to practitioners with varying backgrounds (i.e., those with extensive cognitive behavioral therapy [CBT] training and those without any CBT training [7]) and across cultures all over the world [19]. It has also been modified for many uses, including as a preventative intervention [20] and as a PTSD treatment delivered in a primary care setting [21]. PE provides a model for PTSD treatment that is both straightforward and elegant in its application. Both providers and patients can quickly grasp the model and effectively apply it to individual patient presentations. Thus, PE is individualized and flexible enough to allow use across many patients and settings.

While support for ET is strong, only a few specific randomized trials of PE with Veterans have been completed. Schnurr et al. conducted one of the largest randomized trials of PE with Veterans. Among a sample of female Veterans, they found greater reduction of PTSD in PE than present-centered therapy (PCT), a supportive intervention used to control for the nonspecific effects of psychotherapy [5]. Significant reductions in depression were also apparent. Of note, the reductions in interviewer-rated PTSD symptoms (within group, completers: $d=1.15$ [5]) were somewhat smaller than previous randomized trials in PTSD (within group, completers: $d=$ 3.31 [7]; within group, completers: $d=1.92$ [22]). Bradley et al. and others have suggested that this may reflect a resistance to treatment effect among Veterans. Consistent with this assertion, a meta-analysis of CBT for PTSD found a significantly smaller effect size (within group, $d=$ 0.8 ) in Veterans than in non-Veterans (within group, $d=$ 1.82) for sexual assault [23]. While an effect size of 0.8 demonstrates a large and clinically significant reduction in PTSD, it also suggests that there may be factors specific to Veterans that result in more variability in treatment response than people in the community who have experienced trauma. Despite this variability, PE is clearly an effective treatment for PTSD, associated with large reductions in PTSD and related psychopathology.
Research to date disconfirms the idea that multiple traumatic experiences (as is evident with most Veterans with PTSD) mediate response to PE. Importantly, while many of the patients treated in previous outcome studies had single-incident traumas that were the focus of treatment, multiple-incident traumas have also been included, primarily child sexual abuse (CSA). Indeed, a modification of PE specifically for the treatment of CSA demonstrated excellent results with reductions in PTSD and anxiety greater than PCT and a waitlist [24]. Further, a majority of trauma survivors in PTSD psychotherapy studies to date who sought treatment based on a single incident-trauma also had a history of other traumatic experiences [7,22,24]. In one large study, Foa et al. indicated that "almost all" participants reported at least one other traumatic experience in addition to the target event and 80 percent of participants reported at least one additional incident of interpersonal violence [7]. In another trial, 65 percent of the participants had experienced more than one type of trauma [25]. Thus, significant previous research supports the use of $\mathrm{PE}$ for multiple-incident trauma, such as combat. Additional data from ongoing trials will more precisely examine this issue in military and Veteran populations.

Comorbidity is the rule, not the exception, in PTSD. Some providers report concerns about using PE in the presence of significant comorbidities. This concern may arise from a belief that efficacy studies have restrictive inclusion and exclusion criteria that do not reflect realworld patients [5,7,22,25-26]. However, in the case of the primary randomized control trials for PE, a majority of excluded patients in these studies were excluded based on clinical factors that would supersede PTSD as the primary presenting problem (i.e., imminent suicide risk or current untreated psychotic and/or manic episode). With the exception of severe substance abuse and/or dependence, many common comorbid conditions are well represented in clinical trials of PE. Examination of the samples reveals high rates of comorbid depression (about $40 \%-60 \%$ with current major depressive episode), other anxiety disorders (10\%-50\%) [5,7,22,25], coexisting medical diagnoses [5], and dissociative symptoms $[25,27]$. Further, PE was associated with significant reductions in comorbid depressive and anxiety symptoms (within group, $d=0.59-1.77$ ) $[5,7,26]$. PE has demonstrated significant reductions in reported negative health perceptions and improvement in general social functioning than a waitlist control group [28]. Studies are underway 
examining versions of $\mathrm{PE}$ for patients with comorbid substance dependence and/or abuse, with encouraging preliminary results [29-33]. Indeed, in one large clinical trial that is underway using PE in the comorbid PTSD and substance dependence population, Coffey et al. ${ }^{*}$ are finding a 70 percent reduction in PTSD with PE and 80 to 90 percent abstinence at a 6-month follow-up. Foa and Yusko reported similar outcomes for a study with PTSD and alcohol dependence comorbidity [31]. In a review of the literature on treatment of comorbid substance abuse and PTSD, Henslee and Coffey provide empirically based specific suggestions for the use of PE as part of a residential substance abuse and PTSD program [34]. Thus, people with PTSD and comorbid conditions appear to benefit from PE, both for PTSD symptoms and comorbid conditions.

Another specific comorbidity that has gained attention based on injuries from the conflicts in Afghanistan and Iraq is prolonged postconcussive syndrome (PPCS), also called TBI. While review of this concept is beyond the scope of the current article, preliminary data and the conclusions of the VA/DOD expert consensus panel support the use of PE for the treatment of PTSD among this population [11]. Indeed, the relative simplicity and flexibility of the protocol and the individualized nature of the treatment (e.g., meeting with the Veteran regarding where he or she is as far as cognitive function and areas of difficulty) suggest that PE may be especially well suited for adaptation to comorbid presentations such as PPCS. Studies are currently underway to more closely examine the effect of PPCS on response to PE.

Because of the conflicts in Iraq and Afghanistan, both the Veterans Health Administration (VHA) and DOD are now investing significant resources into training mental health providers in empirically supported treatments for PTSD [35]. As a result, the use of PE among these populations has increased exponentially, resulting in significant relevant clinical field data. While these clinical data have inherent weaknesses (i.e., nonrandomized allocation to treatment, may not represent the full population of Veterans with PTSD, treatment is not closely controlled and monitored, outcomes tend to be self-report), they provide relevant insight into the effec-

* Coffey, Scott. (Division of Psychology, University of Mississippi Medical Center, Jackson, MS). Email to: Sheila Rauch (VA Ann Arbor Healthcare System, Ann Arbor, MI). 2012 Jan 28. tiveness of PE in the clinical Veteran population under natural treatment conditions. To date, these effectiveness data from VA clinics using PE have strongly supported the use of PE among Veterans from all eras. In a large sample of Veterans from the conflicts in Iraq and Afghanistan, Tuerk et al. found significant reductions in selfreported PTSD symptoms with effect size estimates similar to the published randomized trials (within group, intent to treat: $d=1.19$; completers: $d=2.1$ [36]). Significant reductions were also apparent in depressive symptoms. Of note, examination of several patient level factors (i.e., age, race, sex, and service-connected disability) did not find that these factors influenced treatment response or outcome. Additional effectiveness data have also supported the use of PE in specialized VA PTSD programs, including data supporting the effectiveness of PE delivered by telemental health videoconference technology [37-38]. Finally, data collected on training cases within the national PE training initiative also show that the intervention itself has been associated with clinically significant mean improvements of 20 points (completers, within group, $d=1.36$ ) on the PTSD Checklist [39-40]. Additional randomized trials are ongoing in Veteran samples to more closely examine the efficacy and effectiveness of PE and possible factors that may influence outcome.

While PE is perceived by some therapists as an emotionally challenging form of treatment for patients, data from many clinical trials conducted to date demonstrate that it is as well tolerated as other PTSD treatments. In a meta-analysis of dropouts from 25 studies using various forms of CBT for PTSD, Hembree et al. found that the dropout rate from PE alone was 20.5 percent compared with 22.1 percent for anxiety management or CBT alone and 26.9 percent for combination therapies [41]. Rates of dropout from medication trials for PTSD (sertraline, paroxetine, and fluoxetine) range from 22 [42] to 38 percent [43]. Thus, PE is at least as tolerable for patients as other forms of CBT and selective serotonin reuptake inhibitors (SSRIs) for PTSD. Looking specifically at treatment studies with Veterans, published trials report rates of dropout from PE (or ET) ranging between 20 and 35 percent $[4-5,44]$.

Another misconception about PE focuses on a belief that patients get worse before they get better. While this may be the case for some patients, it is not the normative response to engaging in PE. Indeed, many patients show a reduction that starts in the first session and continues 
throughout the program. Tuerk et al. report a pattern of symptom reduction in PE with Veterans that includes accelerated reduction in beginning of treatment followed by continued improvement "at a slower pace" [36]. Foa et al. examined symptom exacerbation in PE and found that only a minority of patients experienced reliable exacerbation (10.5\% for PTSD severity) [45]. Even among patients who had exacerbation as defined in this article, symptom levels did not increase above pretreatment levels. Of importance, exacerbation was not related to increased dropout or reduced treatment response. As such, concern for symptom exacerbation should not prevent the use of $\mathrm{PE}$.

\section{DISSEMINATION OF PROLONGED EXPOSURE: AN ONGOING PROCESS}

Passive dissemination of information about effective treatments and conventional training programs are ineffective in dissemination of effective treatments for PTSD and other mental health problems [46]. However, more interactive training in $\mathrm{PE}$ accompanied by posttraining supervision can be effective. Research has shown that rape crisis counselors [7], Israeli mental health practitioners [47], and VHA mental health providers [5] can effectively deliver PE when trained in this way. For example, when trained using a 5-day workshop and supervised on a regular basis, master's degree social work or counseling clinicians who worked in a community clinic for rape survivors with no prior experience utilizing CBT achieved patient outcomes that matched or exceeded those of experienced CBT therapists [7].

Large-scale training initiatives in PE and other evidence-based treatments have now been implemented within the VA and DOD $[35,46]$. The PE program has involved both intensive training for practitioners and complementary system changes intended to reduce obstacles to treatment delivery [35]. Criteria for successful completion of training include participation in a 4-day interactive workshop followed by completion of a minimum of two PE cases as approved by trained and selected PE consultants who provide weekly detailed consultation and feedback of each case. Staff at the National Center for PTSD worked closely with Dr. Edna Foa, the developer of PE, and Dr. Elizabeth Hembree of the University of Pennsylvania to design and implement the training methodology on a national scale. To date, more than
1,300 VA mental health professionals have attended the workshop as part of the formal implementation program $[40,48]$; within the DOD, similar training services delivered by the Center for Deployment Psychology are underway. Evaluation data from the VA PE Mental Health Training Initiative indicate that the trainings are effective in reducing attitudinal barriers to PE delivery and in increasing clinician self-efficacy for administration of the treatment $[40,48]$.

Within VA, in recognition of the challenges associated with achieving successful practice change by large numbers of providers, training activities have been supplemented by supportive systems interventions. Perhaps most importantly, VHA policy as established in the VHA Handbook 1160.01, Uniform Mental Health Services in VA Medical Centers and Clinics, now specifies that all Veterans with PTSD must have access to evidence-based PTSD treatment (PE or cognitive processing therapy). Other system changes include creation of a standardized progress note template for $\mathrm{PE}$ that is embedded within VA's electronic medical record system, and designation of Evidence-Based Psychotherapy Coordinators at each medical facility whose role is to actively facilitate implementation and delivery of PE and other selected interventions. An attempt has been made to establish permanent capacity within VA to implement $\mathrm{PE}$ treatment by developing training and consultation expertise within a large cadre of VA mental health professionals who are distributed across VHA's 21 service regions. To date, 16 trainers and $70 \mathrm{PE}$ consultants have been selected and trained across the healthcare system $[40,48]$. As training moves to a decentralized model, these VHA experts are in place to ensure a sustained implementation of $\mathrm{PE}$ in future years.

Evaluation of training indicates that clinicians respond very favorably to training in PE. Their pretraining concerns about the intervention are significantly reduced as they learn more about the treatment through participation in the 4-day training and consultation. Clinicians report decreased concern about the emotional and time commitments in providing a trauma-focused treatment in $\mathrm{PE}$ and increased confidence in their ability to implement PE after training. These shifts in attitude are particularly notable upon completion of the consultation process, which clinicians note is highly salient to their learning and continued implementation of PE. At the end of training, approximately 85 percent of the 822 that have completed consultation to date indicate an intention to 
use PE in their routine practice [40,48]. Furthermore, at postconsultation when clinicians were asked about the consultation process, the majority of these clinicians (98\%) reported that they found the consultation process helpful and also indicated that it helped increase their self-confidence in their delivery of PE $[40,48]$.

Although some clinicians have reported challenges to PE implementation, many VA sites have now successfully implemented the treatment. For instance, some clinics have had difficulty implementing PE because of the 90-minute sessions that are required and their high patient volume, though most clinics have overcome this problem. Allowing providers to have longer sessions reduces the number of unique patients that providers can see. However, given the highly cost-effective nature of $\mathrm{PE}$, this may represent only a short-term reduction in capacity: clinicians may, in fact, be able to increase total number of patients seen as PTSD patients complete treatment after a specific number of sessions with notable improvement rather than engaging in continuous longterm care that does not have a defined end or goal. Indeed, data on long-term PE outcomes (out to 10 years posttreatment) supports maintenance of gains [49]. As clinicians are using $\mathrm{PE}$, they often report feeling empowered to help their patients move through care to discharge. Further, PE providers report seeing improvement and recovery as the normative outcomes.

\section{DISCUSSION}

Clinicians and researchers continue to work to improve methods to engage PTSD patients in PE and retain patients in care, including the use of educational materials on PE as a routine part of PTSD clinic intake procedures. Within the VA and DOD, both provider and patient videos have been developed to serve this purpose. These videos anticipate and address obstacles by showing patients and providers describing their firsthand experiences with the treatments. Many clinics have also implemented routine therapy contracts that set expectations for the patient and the provider regarding what is expected of them as they work through the treatment protocol (i.e., compliance with homework, session attendance, access to the therapist between sessions). In some clinics, possible challenges to treatment are discussed with patients prior to beginning treatment. In this way, challenges such as child care, work schedules, low motivation, and unsupportive families can be identified and addressed prior to treatment to minimize dropout and extension of treatment because of missed appointments. For providers, program support assistants can ensure that their time is appropriately blocked and can also track availability for starting new cases based on status of the current cases. To further assist providers, the PE Initiative has implemented drop-in consultation hours for those providers who complete the training requirements. These calls occur several times a week and allow providers to have ongoing clinical and implementation support from other PE providers and national experts in PE.

Many clinical treatment trials, including PE, are ongoing in military, Veteran, and other trauma-exposed groups with PTSD, which will continue to inform optimization of PE treatment. These trials, as well as additional research in clinic settings, include populations that have previously been ruled out of PE studies (i.e., active suicidal risk [50-51], substance dependence [31,34], psychotic disorder [52-53]). Such studies will continue to close the gap as we learn how to apply PE to patients with these severe comorbidities who experience PTSD. In addition, trials examining modifications to optimize the efficiency of PE are also underway. These trials include studies designed to improve stimulus presentations through the use of virtual reality, trials of medications that augment the learning process (such as Dcycloserine), and combination treatment trials (such as combined SSRI and PE treatment). In addition, studies examining how PE works in the brain and body can be used to optimize learning. Through close examination of the mechanisms involved in treatment change, we can learn how to most efficiently provide effective treatment for PTSD. For an update of emotional processing theory and review of mechanism research focused on PE, see Rauch and Foa [13].

Of importance, PE has been subjected to significant empirical scrutiny and has demonstrated efficacy and safety even for complex and comorbid patients. Based on a review of adverse events across all PTSD trials conducted at the Center for the Treatment and Study of Anxiety involving hundreds of patients to date, Dr. Foa ${ }^{*}$ reported that there have been no reported suicide attempts or completed suicides.

\footnotetext{
*Foa, Edna B. (University of Pennsylvania, Philadelphia, PA). Conversation with and email to: Sheila Rauch (VA Ann Arbor Healthcare System, Ann Arbor, MI). 2011 Nov 16.
} 


\section{CONCLUSIONS}

PE is a gold standard treatment for PTSD that has been subjected to many clinical trials supporting its effectiveness in reducing PTSD even among complex and comorbid patients. Thus, if PTSD is primary for a patient and the patient is not in imminent risk of harm (from self or others), PE may be indicated and should be considered as a critical part of the treatment plan. With the dissemination efforts of VA and DOD as well as other community training, clinicians are now able to receive highquality training in the use of $\mathrm{PE}$, making this treatment more widely available than ever before to address the needs of returning Veterans from the conflicts in Iraq and Afghanistan, previous era Veterans, and all trauma survivors.

\section{ACKNOWLEDGMENTS}

\section{Author Contributions:}

Drafting of manuscript: S. A. Rauch, A. Eftekhari, J. I. Ruzek. Critical revision of manuscript for important intellectual content: S. A. Rauch, A. Eftekhari, J. I. Ruzek.

Financial Contributions: The authors have declared that no competing interests exist.

Funding/Support: This article was based on work supported by a Career Development Award (CDA-2) to Dr. Rauch from the VA Office of Research and Development Clinical Sciences Research and Development Service.

\section{REFERENCES}

1. Rothbaum BO, Meadows EA, Resick P, Foy DW. Cognitive-behavioral therapy. In: Foa EB, Keane TM, Friedman MJ, editors. Effective treatments for PTSD: Practice guidelines from the International Society for Traumatic Stress Studies. New York (NY): Guilford Press; 2000. p. 320-25.

2. Keane TM, Fairbank JA, Caddell JM, Zimering RT. Implosive (flooding) therapy reduces symptoms of PTSD in Vietnam combat veterans. Behav Ther. 1989;20(2):245-60. http://dx.doi.org/10.1016/S0005-7894(89)80072-3

3. Boudewyns PA, Hyer L. Physiological response to combat memories and preliminary treatment outcome in Vietnam veteran PTSD patients treated with direct therapeutic exposure. Behav Ther. 1990;21:63-87.

http://dx.doi.org/10.1016/S0005-7894(05)80189-3

4. Glynn SM, Eth S, Randolph ET, Foy DW, Urbaitis M, Boxer L, Paz GG, Leong GB, Firman G, Salk JD, Katzman JW, Crothers J. A test of behavioral family therapy to augment exposure for combat-related posttraumatic stress dis- order. J Consult Clin Psychol. 1999;67(2):243-51.

[PMID:10224735]

http://dx.doi.org/10.1037/0022-006X.67.2.243

5. Schnurr PP, Friedman MJ, Engel CC, Foa EB, Shea MT, Chow BK, Resick PA, Thurston V, Orsillo SM, Haug R, Turner C, Bernardy N. Cognitive behavioral therapy for posttraumatic stress disorder in women: A randomized controlled trial. JAMA. 2007;297(8):820-30.

[PMID:17327524]

http://dx.doi.org/10.1001/jama.297.8.820

6. Cahill SP, Rauch SA, Hembree EA, Foa EB. Effect of cognitive-behavioral treatments for PTSD on anger. J Cogn Psychother. 2003;17(2):113-31. http://dx.doi.org/10.1891/jcop.17.2.113.57434

7. Foa EB, Hembree EA, Cahill SP, Rauch SA, Riggs DS, Feeny NC, Yadin E. Randomized trial of prolonged exposure for posttraumatic stress disorder with and without cognitive restructuring: Outcome at academic and community clinics. J Consult Clin Psychol. 2005;73(5):953-64. [PMID:16287395] http://dx.doi.org/10.1037/0022-006X.73.5.953

8. National Institute for Clinical Excellence. Post-traumatic stress disorder (PTSD): The management of PTSD in adults and children in primary and secondary care. London (England): National Institute for Clinical Excellence; 2005.

9. Foa EB, Keane TM, Friedman MJ, Cohen JA. Effective treatments for PTSD: Practice guidelines from the International Society for Traumatic Stress Studies. 2nd ed. New York (NY): Guilford Press; 2009.

10. Institute of Medicine. Treatment of PTSD: An assessment of the evidence. Washington (DC): Institute of Medicine; 2007. p. 84.

11. National Center for PTSD. Report of (VA) consensus conference: Practice recommendations for the treatment of veterans with comorbid TBI, pain, and PTSD [Internet]. Washington (DC): National Center for PTSD; 2010 [2010 Jan 20]. Available from: http://www.ptsd.va.gov/ professional/pages/handouts-pdf/ TBI PTSD Pain Practice Recommend.pdf

12. Foa EB, Kozak MJ. Emotional processing of fear: Exposure to corrective information. Psychol Bull. 1986;99(1): 20-35. [PMID:2871574]

http://dx.doi.org/10.1037/0033-2909.99.1.20

13. Rauch S, Foa E. Emotional Processing Theory (EPT) and exposure therapy for PTSD. J Contemp Psychother. 2006; 36(2):61-65. http://dx.doi.org/10.1007/s10879-006-9008-y

14. Foa EB, Riggs DS. Posttraumatic stress disorder following assault: Theoretical considerations and empirical findings. Curr Dir Psychol Sci. 1995;4(2):61-65. http://dx.doi.org/10.1111/1467-8721.ep10771786

15. Foa EB, Rauch SA. Cognitive changes during prolonged exposure versus prolonged exposure plus cognitive restructuring in female assault survivors with posttraumatic stress disorder. J Consult Clin Psychol. 2004;72(5):879-84. 


\section{[PMID:15482045]}

http://dx.doi.org/10.1037/0022-006X.72.5.879

16. Chaplin EW, Levine BA. The effects of total exposure duration and interrupted versus continuous exposure in flooding therapy. Behav Ther. 1981;12(3):360-68. http://dx.doi.org/10.1016/S0005-7894(81)80124-4

17. Rauch SA, Foa EB, Furr JM, Filip JC. Imagery vividness and perceived anxious arousal in prolonged exposure treatment for PTSD. J Trauma Stress. 2004;17(6):461-65. [PMID:15730064] http://dx.doi.org/10.1007/s10960-004-5794-8

18. Van Minnen A, Hagenaars M. Fear activation and habituation patterns as early process predictors of response to prolonged exposure treatment in PTSD. J Trauma Stress. 2002;15(5):359-67. [PMID:12392223] http://dx.doi.org/10.1023/A:1020177023209

19. Foa EB. Disseminating evidence-based treatments within systems and across countries: Lessons learned from prolonged exposure therapy. 31st Annual Conference of the Anxiety Disorders Association of America; 2011 Oct 24; New Orleans, LA.

20. Foa EB, Keane TM, Friedman MJ. Effective treatments for PTSD: Practice guidelines from the International Society for Traumatic Stress Studies. New York (NY): Guilford Press; 2000.

21. Cigrang JA, Rauch SA, Avila LL, Bryan CJ, Goodie JL, Hryshko-Mullen A, Peterson AL. Treatment of active-duty military with PTSD in primary care: Early findings. Psychol Serv. 2011;8(2):104-13. http://dx.doi.org/10.1037/a0022740

22. Foa EB, Dancu CV, Hembree EA, Jaycox LH, Meadows EA, Street GP. A comparison of exposure therapy, stress inoculation training, and their combination for reducing posttraumatic stress disorder in female assault victims. J Consult Clin Psychol. 1999;67(2):194-200.

[PMID:10224729]

http://dx.doi.org/10.1037/0022-006X.67.2.194

23. Bradley R, Greene J, Russ E, Dutra L, Westen D. A multidimensional meta-analysis of psychotherapy for PTSD. Am J Psychiatry. 2005;162(2):214-27. [PMID:15677582] http://dx.doi.org/10.1176/appi.ajp.162.2.214

24. McDonagh A, Friedman M, McHugo G, Ford J, Sengupta A, Mueser K, Demment CC, Fournier D, Schnurr PP, Descamps M. Randomized trial of cognitive-behavioral therapy for chronic posttraumatic stress disorder in adult female survivors of childhood sexual abuse. J Consult Clin Psychol. 2005;73(3):515-24. [PMID:15982149] http://dx.doi.org/10.1037/0022-006X.73.3.515

25. Taylor S, Thordarson DS, Maxfield L, Fedoroff IC, Lovell K, Ogrodniczuk J. Comparative efficacy, speed, and adverse effects of three PTSD treatments: Exposure therapy, EMDR, and relaxation training. J Consult Clin Psychol. 2003;71(2):330-38. [PMID:12699027] http://dx.doi.org/10.1037/0022-006X.71.2.330
26. Rothbaum BO, Astin MC, Marsteller F. Prolonged exposure versus Eye Movement Desensitization and Reprocessing (EMDR) for PTSD rape victims. J Trauma Stress. 2005;18(6):607-16. [PMID:16382428]

http://dx.doi.org/10.1002/jts.20069

27. Hagenaars MA, van Minnen A, Hoogduin KA. The impact of dissociation and depression on the efficacy of prolonged exposure treatment for PTSD. Behav Res Ther. 2010;48(1):19-27. [PMID:19766987] http://dx.doi.org/10.1016/j.brat.2009.09.001

28. Rauch SA, Grunfeld TE, Yadin E, Cahill SP, Hembree E, Foa EB. Changes in reported physical health symptoms and social function with prolonged exposure therapy for chronic posttraumatic stress disorder. Depress Anxiety. 2009; 26(8):732-38. [PMID:18781660] http://dx.doi.org/10.1002/da.20518

29. Coffey SF, Stasiewicz PR, Hunt Y, Linkovich-Kyle TL. Readiness to change as a predictor of retention in a study of trauma focused on exposure therapy for alcohol dependent PTSD individuals. 39th Annual Convention of the Association for Behavioral and Cognitive Therapies; 2005; Washington, DC.

30. Riggs DS, Pai A, Volpicelli J, Imms P, Foa EB. Drop out from treatment targeting comorbid PTSD and alcohol dependence. 39th Annual Convention of the Association for Behavioral and Cognitive Therapies; 2005; Washington, DC.

31. Foa EB, Yusko DA. Concurrent treatment for alcohol dependence and PTSD. 44th Annual Convention of the Association for Behavioral and Cognitive Therapies; 2010 Nov 18-21; San Francisco, CA.

32. Riggs DS, Foa EB, Stewart SH, Conrod PJ. Treatment for co-morbid posttraumatic stress disorder and substance use disorders. In: Anxiety and substance use disorders: The vicious cycle of comorbidity. New York (NY): Springer Science + Business Media; 2008. p. 119-37.

33. Tuerk P, Brady KT, Grubaugh AL. Clinical case discussion: Combat PTSD and substance use disorders. J Addict Med. 2009;3(4):189-93. [PMID:21769016] http://dx.doi.org/10.1097/ADM.0b013e3181a9d276

34. Henslee AM, Coffey SF. Exposure therapy for posttraumatic stress disorder in a residential substance use treatment facility. Prof Psychol Res Pr. 2010;41(1):34-40. [PMID:20368746] http://dx.doi.org/10.1037/a0018235

35. Karlin BE, Ruzek JI, Chard KM, Eftekhari A, Monson CM, Hembree EA, Resick PA, Foa EB. Dissemination of evidence-based psychological treatments for posttraumatic stress disorder in the Veterans Health Administration. J Trauma Stress. 2010;23(6):663-73. [PMID:21171126] http://dx.doi.org/10.1002/jts.20588

36. Tuerk PW, Yoder M, Grubaugh A, Myrick H, Hamner M, Acierno R. Prolonged exposure therapy for combat-related posttraumatic stress disorder: An examination of treatment effectiveness for veterans of the wars in Afghanistan and 
Iraq. J Anxiety Disord. 2011;25(3):397-403.

[PMID:21131170]

http://dx.doi.org/10.1016/j.janxdis.2010.11.002

37. Rauch SA, Defever E, Favorite T, Duroe A, Garrity C, Martis B, Liberzon I. Prolonged exposure for PTSD in a Veterans Health Administration PTSD clinic. J Trauma Stress. 2009;22(1):60-64. [PMID:19145643] http://dx.doi.org/10.1002/jts.20380

38. Tuerk PW, Yoder M, Ruggiero KJ, Gros DF, Acierno R. A pilot study of prolonged exposure therapy for posttraumatic stress disorder delivered via telehealth technology. J Trauma Stress. 2010;23(1):116-23. [PMID:20135675]

39. Weathers FW, Litz B, Herman D, Huska J, Keane T. The PTSD Checklist (PCL): Reliability, validity, and diagnostic utility. Annual Convention of the International Society for Traumatic Stress Studies; 1993; San Antonio, TX.

40. Eftekhari A, Ruzek JI, Foa EB, et al. Prolonged exposure therapy in VHA. 44th Annual Meeting of the Association for Behavioral and Cognitive Therapies; 2010 Nov 18-21; San Francisco, CA.

41. Hembree EA, Foa EB, Dorfan NM, Street GP, Kowalski J, Tu X. Do patients drop out prematurely from exposure therapy for PTSD? J Trauma Stress. 2003;16(6):555-62. [PMID:14690352] http://dx.doi.org/10.1023/B:JOTS.0000004078.93012.7d

42. Connor KM, Sutherland SM, Tupler LA, Malik ML, Davidson JR. Fluoxetine in post-traumatic stress disorder. Randomised, double-blind study. Br J Psychiatry. 1999; 175:17-22. [PMID:10621763] http://dx.doi.org/10.1192/bjp.175.1.17

43. Tucker P, Zaninelli R, Yehuda R, Ruggiero L, Dillingham $\mathrm{K}$, Pitts CD. Paroxetine in the treatment of chronic posttraumatic stress disorder: Results of a placebo-controlled, flexible-dosage trial. J Clin Psychiatry. 2001;62(11):860-68. [PMID:11775045] http://dx.doi.org/10.4088/JCP.v62n1105

44. Schnurr PP, Friedman MJ, Foy DW, Shea MT, Hsieh FY, Lavori PW, Glynn SM, Wattenberg M, Bernardy NC. Randomized trial of trauma-focused group therapy for posttraumatic stress disorder: Results from a Department of Veterans Affairs cooperative study. Arch Gen Psychiatry. 2003;60(5):481-89. [PMID:12742869] http://dx.doi.org/10.1001/archpsyc.60.5.481

45. Foa EB, Zoellner LA, Feeny NC, Hembree EA, AlvarezConrad J. Does imaginal exposure exacerbate PTSD symptoms? J Consult Clin Psychol. 2002;70(4):1022-28. [PMID:12182265] http://dx.doi.org/10.1037/0022-006X.70.4.1022

46. Ruzek JI, Rosen RC. Disseminating evidence-based treatments for PTSD in organizational settings: A high priority focus area. Behav Res Ther. 2009;47(11):980-89.

[PMID:19632668]

http://dx.doi.org/10.1016/j.brat.2009.07.008
47. Cahill SP, Foa EB, Hembree EA, Marshall RD, Nacash N. Dissemination of exposure therapy in the treatment of posttraumatic stress disorder. J Trauma Stress. 2006;19(5): 597-610. [PMID:17075914] http://dx.doi.org/10.1002/jts.20173

48. Eftekhari A, Ruzek JI, Crowley J, Rosen CS, Gregory G, Karlin BE. Prolonged exposure mental health training initiative: Progress, program evaluation and sustainability. 3rd Annual VA Mental Health Conference; 2011; Baltimore, MD.

49. Resick P. Long term follow-up of a clinical trial of cognitive processing therapy and prolonged exposure therapy. 2010 Annual Trauma Symposium at National Trauma Institute; 2010; San Antonio, TX.

50. Harned MS, Linehan MM. Integrating dialectical behavior therapy and prolonged exposure to treat co-occurring borderline personality disorder and PTSD: Two case studies. Cogn Behav Pract. 2008;15:263-76.

http://dx.doi.org/10.1016/j.cbpra.2007.08.006

51. Harned MS, Jackson SC, Comtois KA, Linehan MM. Dialectical behavior therapy as a precursor to PTSD treatment for suicidal and/or self-injuring women with borderline personality disorder. J Trauma Stress. 2010;23(4):421-29. [PMID:20648564] http://dx.doi.org/10.1002/jts.20553

52. Grubaugh AL, Zinzow HM, Paul L, Egede LE, Frueh BC. Trauma exposure and posttraumatic stress disorder in adults with severe mental illness: A critical review. Clin Psychol Rev. 2011;31(6):883-99. [PMID:21596012] http://dx.doi.org/10.1016/j.cpr.2011.04.003

53. Frueh BC, Grubaugh AL, Cusack KJ, Kimble MO, Elhai JD, Knapp RG. Exposure-based cognitive-behavioral treatment of PTSD in adults with schizophrenia or schizoaffective disorder: A pilot study. J Anxiety Disord. 2009;23(5): 665-75. [PMID:19342194] http://dx.doi.org/10.1016/j.janxdis.2009.02.005

Submitted for publication August 29, 2011. Accepted in revised form January 23, 2012.

This article and any supplementary material should be cited as follows:

Rauch SA, Eftekhari A, Ruzek JI. Review of exposure therapy: A gold standard for PTSD treatment. J Rehabil Res Dev. 2012;49(5):679-88. http://dx.doi.org/10.1682/JRRD.2011.08.0152

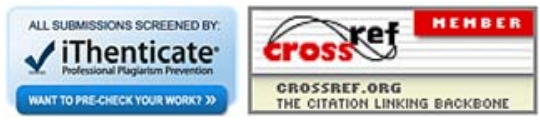


